TITLE:

\title{
Effects of the carrier-envelope phase in the multiphoton ionization regime
}

AUTHOR(S):

Nakajima, T; Watanabe, S

CITATION:

Nakajima, T ...[et al]. Effects of the carrier-envelope phase in the multiphoton ionization regime. Physical Review Letters 2006, 96(21): 213001.

ISSUE DATE:

2006-06-02

URL:

http://hdl.handle.net/2433/50427

RIGHT:

Copyright 2006 American Physical Society 


\title{
Effects of the Carrier-Envelope Phase in the Multiphoton Ionization Regime
}

\author{
Takashi Nakajima ${ }^{1,2, *}$ and Shuntaro Watanabe ${ }^{2}$ \\ ${ }^{1}$ Institute of Advanced Energy, Kyoto University, Gokasho, Uji, Kyoto 611-0011, Japan \\ ${ }^{2}$ Institute for Solid State Physics, The University of Tokyo, 5-1-5 Kashiwanoha, Kashiwa, Chiba 277-8581, Japan
}

(Received 11 August 2005; published 2 June 2006)

\begin{abstract}
We theoretically investigate the effects of the carrier-envelope phase of few-cycle laser pulses in the multiphoton ionization regime. For atoms with low ionization potential, total ionization yield barely exhibits phase dependence, as expected. However, population of some bound states clearly shows phase dependence. This implies that the measurement of the carrier-envelope phase would be possible through the photoemission between bound states without energy-and-angle-resolved photoelectron detection. The considered scheme could be particularly useful to measure the carrier-envelope phase for a light source without an amplifier, such as a laser oscillator, which cannot provide sufficient pulse energy to induce tunneling ionization.
\end{abstract}

Because of the rapid advances of laser technology and strong field physics in the last few years, it is now well established that an intense few-cycle pulse induces various effects with dependences on the carrier-envelope phase (CEP) in terms of photoionization and high harmonic generation in the strong field regime [1-9]. The physical picture that is most conveniently employed is tunneling ionization, and experimental results are well explained with this model. Based on the phase-dependent tunneling ionization, CEP has been successfully measured by the energy-and-angle-resolved photoelectron detection. The tunneling picture, however, does not tell us anything for the case in which the field is rather weak to induce tunneling ionization. It does not tell us anything about the bound state population, either.

In this Letter we theoretically show that the CEPdependent effects can be clearly seen with Cs atom for nearly single-cycle pulses $[10,11]$, even in the multiphoton ionization regime without energy-and-angle-resolved photoelectron detection. We would like to emphasize that the phase-dependent effects we report in this Letter are different from those in the literature in a few important aspects: first, the intensity range we consider here is so-called multiphoton ionization regime where tunneling ionization hardly takes place. Indeed, the ionization yield obtained from the ADK theory [12] in the intensity range of our interest is more than several orders of magnitude smaller than that we obtain from the solution of time-dependent Schrödinger equation. Second, the CEP dependence we have found is in the bound state population rather than the ionization or photoelectron yields. Third, we have seen the effects with Cs atom, which has much lower ionization potential compared with hydrogen or rare gas atoms that are most commonly used for the study of phase-dependent effects. Summarizing the above features, the use of atoms with low ionization potential enables us to observe the phase-dependent population in some bound states through the photoemission detection, which is technically much simpler than the energy-and-angle-resolved photoelectron detection, at considerably low intensities ever explored.

In Fig. 1 we show the level scheme of Cs atom. Cs atoms in the $6 s$ ground state interact with a few-cycle laser pulse in the near-infrared wavelength. In order to describe the time-dependent dynamics of the $\mathrm{Cs}$ atom, we solve the three-dimensional time-dependent Schrödinger equation,

$$
i \frac{\partial \Psi(t)}{\partial t}=\left[H_{0}+V(t)\right] \Psi(t),
$$

where $H_{0}$ is the field-free atomic Hamiltonian of Cs and $V(t)$ the time-dependent interaction between the atom and the laser field which is defined by $V(t)=-\hat{\boldsymbol{\epsilon}} \cdot \boldsymbol{r} E(t)$ with $E(t)$ being the electric field, $\hat{\boldsymbol{\epsilon}}$ the polarization vector which is assumed to be linear throughout this Letter, and $\boldsymbol{r}$ the position operator of the valence electron. Because of the reason we will explain below, the electric field, $E(t)$, is defined via the vector potential, $A(t)$. Specifically we assume that the vector potential has a Gaussian temporal envelope, i.e.,

$$
A(t)=\hat{\boldsymbol{\epsilon}} A_{0} \exp \left[-4 \ln 2\left(\frac{\omega t}{2 \pi N}\right)^{2}\right] \sin (\omega t+\phi),
$$

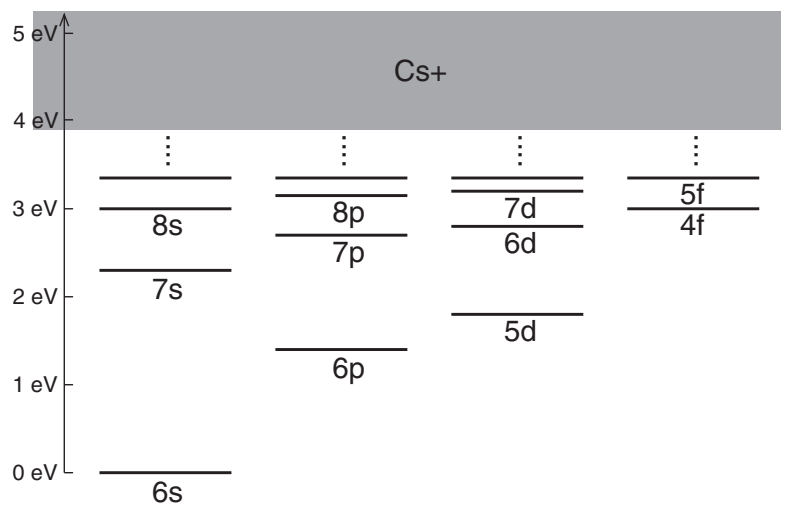

FIG. 1. Level scheme of Cs. 
where $\phi$ is the CEP, $A_{0}$ the envelope of the vector potential, and $\omega$ the photon energy. $N$ is the number of cycles for the full width at half maximum (FWHM) of the amplitude of vector potential. Note that $N$ can be a noninteger number. Using the vector potential the electric field is derived as $E(t)=-\partial A(t) / \partial t$. The definition of the electric field via the vector potential is crucial for few-cycle pulses in order to guarantee that the vector potential, $A(t)$, vanishes at $t=$ $\pm \infty$, or equivalently the pulse area under the $E(t)$ function becomes exactly zero after integrating $E(t)$ over the entire pulse duration [13]. Otherwise unphysical results will be obtained.

Now, the total wave function, $\Psi(t)$, is expanded on atomic basis functions constructed in a spherical box of 200-500 a.u. Since the Cs atom has only one valence electron in the outermost shell and the ionization potential of $\mathrm{Cs}^{+}$lies $25.1 \mathrm{eV}$ above the ionization potential of Cs, it is safe to assume that the core excitation does not take place in the intensity range we consider in this Letter. This means that only the valence electron will play a role during the interaction, justifying the use of a pseudopotential to effectively accounts for the interaction of the valence electron with the core electrons $[14,15]$. The basis functions for the valence electron, $\phi_{n l m}(\boldsymbol{r})$, can be decomposed into the product of the radial functions, $P_{n l}(r)$, and the spherical harmonics, $Y_{l m}(\theta, \phi)$, for the states given by a set of principal, angular, and magnetic quantum numbers, $(n, l, m)$ :

$$
\phi_{n l m}(\boldsymbol{r})=\frac{P_{n l}(r)}{r} Y_{l m}(\theta, \phi),
$$

where $P_{n l}(r)$ satisfies the Schrödinger equation,

$$
\left[\frac{1}{2} \frac{d^{2}}{d r^{2}}+\left(E_{n l}+V_{l}(r)-\frac{l(l+1)}{2 r^{2}}\right)\right] P_{n l}(r)=0 .
$$

In the above equation, $E_{n l}$ is the eigenenergy and $V_{l}(r)$ the pseudopotential. For the numerical results presented in this

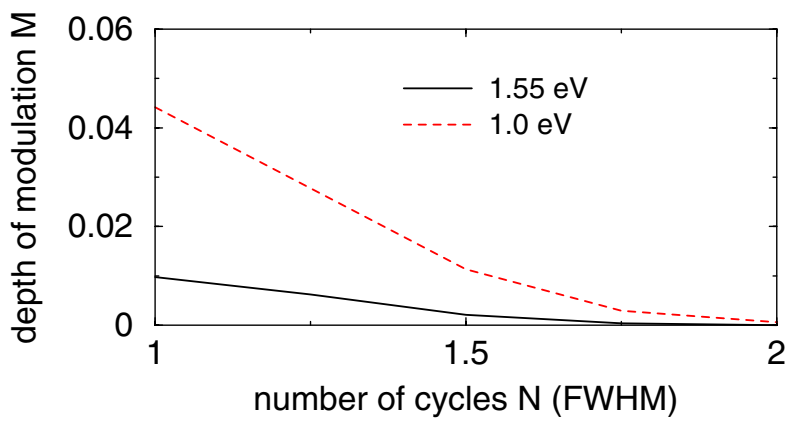

FIG. 2 (color online). Depth of modulation, $M$, for $\mathrm{Cs}$ as a function of the number of cycles, $N$, for the photon energy of $1.55 \mathrm{eV}$ (solid line) at the peak intensity of $10^{11} \mathrm{~W} / \mathrm{cm}^{2}$, and for the photon energy of $1.0 \mathrm{eV}$ (dashed line) at the peak intensity of $4.2 \times 10^{10} \mathrm{~W} / \mathrm{cm}^{2}$. Note that both curves correspond to the same Keldysh parameter, $\gamma=18$. For the definition of $M$, see Eq. (5).
Letter, photon energy is assumed to be $1.55 \mathrm{eV}(1.0 \mathrm{eV})$ and the peak intensity is chosen to be $10^{11} \mathrm{~W} / \mathrm{cm}^{2}(4.2 \times$ $10^{10} \mathrm{~W} / \mathrm{cm}^{2}$ ). Note that the Keldysh parameter, $\gamma$, is 18 for both cases.

To start with, we examine the CEP dependence of the total ionization yield, $P$, which is in principle a function of the phase, $\phi$. We define the depth of modulation, $M$, in the total ionization signal as

$$
M=\frac{P\left(\phi_{\max }\right)-P\left(\phi_{\min }\right)}{\frac{1}{2}\left[P\left(\phi_{\max }\right)+P\left(\phi_{\min }\right)\right]},
$$

where $\phi_{\max }\left(\phi_{\min }\right)$ is the CEP which gives the maximum (minimum) total ionization yield. In Fig. 2 we present the variation of $M$ as a function of the number of cycles, $N$, for the photon energy of $1.55 \mathrm{eV}$. It can be seen that the value of $M$ itself is very small, as expected, since ionization is a very low order process for this atom at this photon energy, and moreover, we are looking at the depth of modulation of the total ionization yield rather than the angle-and-energyresolved photoelectrons. For comparison, Fig. 2 also shows the result for the photon energy of $1.0 \mathrm{eV}$ at the intensity of $4.2 \times 10^{10} \mathrm{~W} / \mathrm{cm}^{2}$. Clearly, for a given $N$, the value of $M$ is a little bit larger when the photon energy becomes smaller, because smaller photons require photoionization processes that are of higher order.

When we look at the population of bound states, however, the situation is quite different. A representative result is shown in Fig. 3 for the pulse with $N=1$. Significant CEP dependence is found for the $5 d, 8 p$, and $4 f$ states, while other states which have much more population barely exhibit CEP dependence. For comparison, we have further performed the calculation without ionization,
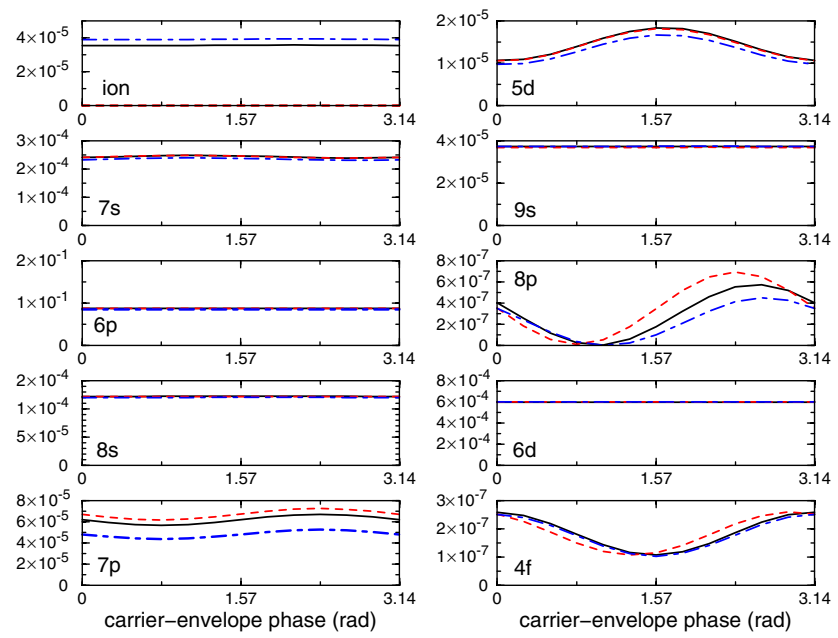

FIG. 3 (color online). Total ionization yield and the population of some bound states of $\mathrm{Cs}$ as a function of carrier-envelope phase for $1.55 \mathrm{eV}$ photon and the peak intensity of $10^{11} \mathrm{~W} / \mathrm{cm}^{2}$ with $N=1$. The calculated result without ionization are shown by the dashed line in each panel. The calculated result with ionization but using a different pseudopotential [Ref. [16]] is shown by the dot-dashed line in each panel. 
the purpose of which is to examine whether the CEP dependence of the bound state population essentially comes from the ionization process. The results are shown by the dashed line in each panel of Fig. 3. For the $8 p$ and $4 f$ states the calculated modulation turned out to be slightly shifted by removing the continuum. Nevertheless, qualitative behavior is practically the same. For the $5 d$ state the behavior is practically identical with and without ionization. This clearly shows that the CEP-dependent population of the bound states essentially stems from the photoabsorption processes even without any kinds of ionization mechanism. We have further performed calculations including ionization processes but with a different pseudopotential [16] to check the reliability of our theoretical results. The results are shown by the dot-dashed line in each panel of Fig. 3. The agreement is very good, which gives us more confidence in our theoretical results. For the circularly polarized field, however, we have found no phase dependence. Now the next question is whether this phase dependence is robust against fluctuation of intensity and number of cycles. To check this, we have carried out calculations for the photon energy of $1.55 \mathrm{eV}$ at the intensity of $10^{11} \mathrm{~W} / \mathrm{cm}^{2}$ and $N=1$ with $\pm 10 \%$ different intensities and number of cycles. The results are shown in Figs. 4(a) and 4(b) for the population of $5 d, 8 p$, and $4 f$ states. It can be seen that the phase dependence is quite robust against the fluctuation of not only intensity but also number of cycles. To be even more realistic, we have carried out a spatial integration assuming a Gaussian distribution function to take into account the spatial intensity distribution around the laser focus. We have found that the deterioration the modulation contrast is very small upon spatial integration.

What is the origin of the phase-dependent population? To understand the underlying physics, we now consider a three-level ladder system, $|0\rangle,|1\rangle$, and $|2\rangle$ interacting with a few-cycle pulse. To simplify the mathematical expression, we assume that the electric field is linearly polarized and written as $E(t)=\varepsilon_{0}(t) \cos (\omega t+\phi)$, which is equivalent to say that we will discard the term with a time derivative of the field envelope, $\varepsilon_{0}(t)$, in the following equations. Note that this simplification does not spoil the essence of phys-
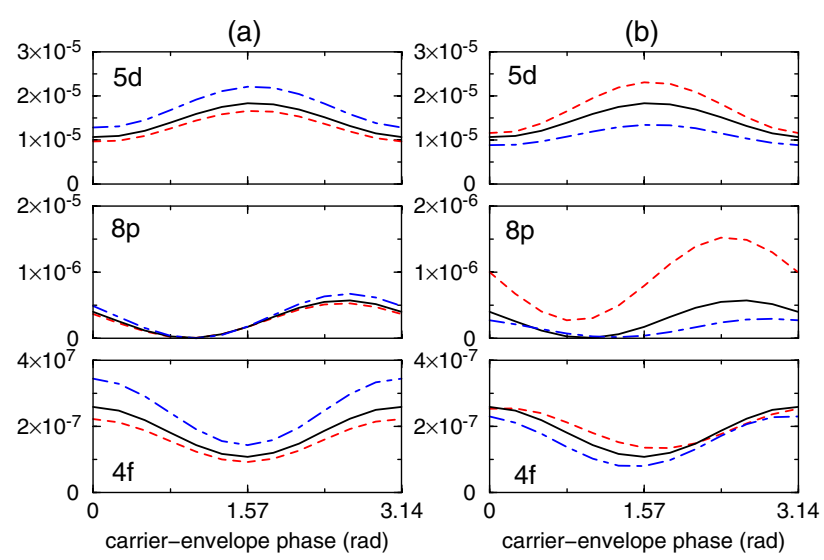

FIG. 4 (color online). Change of modulation for $5 d, 8 p$, and $4 f$ states (a) at slightly different intensities, $I=0.9 \times$ $10^{11} \mathrm{~W} / \mathrm{cm}^{2}$ (dashed line), $10^{11} \mathrm{~W} / \mathrm{cm}^{2}$ (solid line), and $1.1 \times 10^{11} \mathrm{~W} / \mathrm{cm}^{2}$ (dot-dashed line) with $N=1$, and (b) with slightly different number of cycles, $N=0.9$ (dashed line), 1.0 (solid line), and 1.1 (dot-dashed line) at the intensity of $10^{11} \mathrm{~W} / \mathrm{cm}^{2}$. Photon energy is taken to be $1.55 \mathrm{eV}$ for all graphs.

ics, since the purpose of the discussion on the three-level system is to understand the physical origin of the phasedependent population we have seen in Fig. 3 for Cs. Introducing the time-dependent amplitudes as $c_{j}(t)$ for states $|j\rangle(j=0,1,2)$, the amplitude equations read

$$
\begin{aligned}
\dot{c}_{0}(t)= & i \mu_{01} \varepsilon_{0}(t) \cos (\omega t+\phi) e^{-i E_{10} t} c_{1}(t), \\
\dot{c}_{1}(t)= & i \mu_{10} \varepsilon_{0}(t) \cos (\omega t+\phi) e^{i E_{10} t} c_{0}(t) \\
& +i \mu_{12} \varepsilon_{0}(t) \cos (\omega t+\phi) e^{-i E_{21} t} c_{2}(t), \\
\dot{c}_{2}(t) & =i \mu_{21} \varepsilon_{0}(t) \cos (\omega t+\phi) e^{i E_{21} t} c_{1}(t),
\end{aligned}
$$

where $E_{j k}\left(=E_{j}-E_{k}\right)$ and $\mu_{j k}(j, k=0,1,2$ with $j \neq k)$ are the energy difference and the dipole moment between $|j\rangle$ and $|k\rangle$, respectively. Assuming the weak field which implies $c_{0}(t) \sim 1$ for all $t, c_{1}(t)$ and $c_{2}(t)$ can be formally solved by using the first and the second order timedependent perturbation theory. The solutions read

$$
\begin{gathered}
c_{1}(t)=i \mu_{10}\left[e^{i \phi} \int_{-\infty}^{t} \varepsilon_{0}\left(t_{1}\right) e^{i\left(\omega+E_{10}\right) t_{1}} d t_{1}+e^{-i \phi} \int_{-\infty}^{t} \varepsilon_{0}\left(t_{1}\right) e^{-i\left(\omega-E_{10}\right) t_{1}} d t_{1}\right] \\
c_{2}(t)=-\frac{\mu_{21} \mu_{10}}{4}\left[e^{i 2 \phi} \int_{-\infty}^{t} \int_{-\infty}^{t_{1}} \varepsilon_{0}\left(t_{1}\right) \varepsilon_{0}\left(t_{2}\right) e^{i\left(\omega+E_{21}\right) t_{1}} e^{i\left(\omega+E_{10}\right) t_{2}} d t_{1} d t_{2}+\int_{-\infty}^{t} \int_{-\infty}^{t_{1}} \varepsilon_{0}\left(t_{1}\right) \varepsilon_{0}\left(t_{2}\right) e^{i\left(\omega+E_{21}\right) t_{1}} e^{-i\left(\omega-E_{10}\right) t_{2}} d t_{1} d t_{2}\right. \\
\left.+\int_{-\infty}^{t} \int_{-\infty}^{t_{1}} \varepsilon_{0}\left(t_{1}\right) \varepsilon_{0}\left(t_{2}\right) e^{-i\left(\omega-E_{21}\right) t_{1}} e^{i\left(\omega+E_{10}\right) t_{2}} d t_{1} d t_{2}+e^{-i 2 \phi} \int_{-\infty}^{t} \int_{-\infty}^{t_{1}} \varepsilon_{0}\left(t_{1}\right) \varepsilon_{0}\left(t_{2}\right) e^{-i\left(\omega-E_{21}\right) t_{1}} e^{-i\left(\omega-E_{10}\right) t_{2}} d t_{1} d t_{2}\right] .
\end{gathered}
$$

Equation (10) clearly shows that, in principle, not only the resonant (fourth) term but also the antiresonant (first) term and the cross (second and third) terms can contribute to the state amplitude, and therefore population. For long pulses the resonant term usually far dominates over the other, and hence the phase dependence vanishes. For a few-cycle pulse, however, all terms can make a non-negligible contribution because of the extremely short pulse duration. Note that a 
similar argument holds for a three-level $\Lambda$ system. We have verified the above argument by carrying out the timedependent calculations for the three-level system by letting $|0\rangle,|1\rangle$, and $|2\rangle$ to be $6 s, 6 p$ (or $7 p$ or $8 p$ ), and $5 d$ (or $6 d$ or $7 d$ ) of Cs, respectively. Clear phase dependence has been reproduced even for the three-level system. Obviously this argument can be extended to the case of Cs, which explains why we have seen the strong phase dependence in Fig. 3 for the far-off resonant states while the phase dependence disappears for the near-resonant states. It also explains why the phase shifts in Fig. 3 are different for different states: depending on the contribution of each term in Eq. (10), the phase shift and also the depth of modulation can be different. Finally, we note that similar equations can be derived for the case of circular polarization, which explains why there is no phase dependence if the pulse is circularly polarized: there exists only one (resonant) term because of the dipole selection rule in this case, and hence the dynamics do not depend on the phase.

The above theoretical findings suggest that the CEPdependent bound state population of Cs can be utilized for the measurement of the CEP of few-cycle pulses through the photoemission from those states. For example, population of the $4 f$ state is about $10^{-7}$ by the $N=1$ pulse for the $1.55 \mathrm{eV}$ photon and the peak intensity of $10^{11} \mathrm{~W} / \mathrm{cm}^{2}$. If the $\mathrm{Cs}$ vapor is heated to $150^{\circ} \mathrm{C}$, the number densities of $\mathrm{Cs}$ atoms and $\mathrm{Cs}_{2}$ molecules are about $2 \times 10^{14} \mathrm{~cm}^{-3}$ and $2 \times 10^{11} \mathrm{~cm}^{-3}$ [17], respectively. This means that the contribution of $\mathrm{Cs}_{2}$ molecules can be safely neglected. Furthermore, at this Cs vapor temperature, the collisional decay rate of population out of the state is of the order of $10^{5}-10^{6} \mathrm{~s}^{-1}$ [18], implying that the collisional population transfer is negligible in the time scale of the spontaneous decay, which is about $40 \mathrm{~ns}$ and $300 \mathrm{~ns}$ for $4 f$ and $8 p$, respectively. These considerations ensure that, if the gating detection is employed after the pulse, the photoemission signal due to the spontaneous decay precisely reflects population of the bound states. Assuming that the interaction volume is $10^{-3} \mathrm{~cm}^{3}, 2 \times 10^{4}$ atoms are excited to the $4 f$ state, whose population can be monitored from the photoemission at $1002 \mathrm{~nm}$ down to the $5 d$ state. Photoemission from the $8 p$ state down to the $5 d$ state at the wavelength of $802 \mathrm{~nm}$ is another candidate.

In summary, we have theoretically investigated the effects of the carrier-envelope phase of few-cycle pulses for atoms with low ionization potential in the multiphoton ionization regime. Specific results have been presented for the Cs atom. We have found that the population of some bound states exhibits significant phase dependence for the linearly polarized field. In contrast, total ionization yield barely shows phase dependence, as expected. We interpret that the observed phase dependence is due to the competition between the resonant and other (antiresonant as well as cross) interactions. This interpretation is also consistent with our findings that we have seen no phase dependence for the circularly polarized field. Since relatively low intensity $\left(\sim 10^{11} \mathrm{~W} / \mathrm{cm}^{2}\right)$ is sufficient for our scheme with Cs, photoemission detection could be a useful technique to measure the carrier-envelope phase for a light pulse coming out of, say, a laser oscillator without an amplifier. Before closing this Letter, we note that similar and even more significant phase-dependent effects have been found for the hydrogen atom in both bound state population and total ionization yield [19].

T. N. acknowledges useful discussions with Prof. M. Kawasaki and Dr. H. Yamada regarding the coexistence ratio of Cs atoms and $\mathrm{Cs}_{2}$ molecules in a Cs vapor. This work was supported by the Grant in Aid for scientific research from the Ministry of Education and Science of Japan.

*Email address: t-nakajima@iae.kyoto-u.ac.jp

[1] E. Cormier and P. Lambropoulos, Eur. Phys. J. D 2, 15 (1998).

[2] I. P. Christov, Opt. Lett. 24, 1425 (1999).

[3] I. P. Christov, Appl. Phys. B 70, 459 (2000).

[4] P. Dietrich, F. Krausz, and P. B. Corkum, Opt. Lett. 25, 16 (2000).

[5] G. G. Paulus et al., Nature (London) 414, 182 (2001).

[6] G. G. Paulus et al., Phys. Rev. Lett. 91, 253004 (2003).

[7] D. B. Milosevic, G. G. Paulus, and W. Becker, Phys. Rev. Lett. 89, 153001 (2002).

[8] F. Lindner et al., Phys. Rev. Lett. 92, 113001 (2004).

[9] C. Altucci, V. Tosa, R. Velotta, and C. H. Nam, Phys. Rev. A 70, 065402 (2004).

[10] S. Adachi, P. Kumbhakar, and T. Kobayashi, Opt. Lett. 29, 1150 (2004).

[11] K. Yamane et al., Opt. Lett. 28, 2258 (2003).

[12] M. V. Ammosov, N. B. Delone, and V. P. Krainov, Sov. Phys. JETP 64, 1191 (1986).

[13] S. Chelkowski and A.D. Bandrauk, Phys. Rev. A 65, 061802(R) (2002).

[14] W. Nicklich et al., Phys. Rev. Lett. 69, 3455 (1992).

[15] H. Xu, Ph.D. thesis, University of Southern California, 1993.

[16] M. Marinescu, H. R. Sadeghpour, and A. Dalgarno, Phys. Rev. A 49, 982 (1994).

[17] M. A. Bouchiat, J. Guena, Ph. Jacquier, and M. Lintz, Chem. Phys. Lett. 199, 85 (1992).

[18] A. C. Tam, T. Yabuzaki, S. M. Curry, M. Hou, and W. Happer, Phys. Rev. A 17, 1862 (1978).

[19] T. Nakajima and S. Watanabe, Opt. Lett. 31, 1920 (2006). 